\title{
A new mathematical approach for determining kinetic parameters of curing process
}

\author{
Murat Oz, Gamze Tanoğlu, Neslişah Imamoğlu, Yusufcan Uz and Metin Tanoğlu \\ Izmir Institute of Technology, Izmir, Turkey
}

Received: 13 June 2021, Accepted: 16 June 2021

Published online: 12 July 2021.

\begin{abstract}
We proposed a new algorithm (GMN) for determining pre-exponential and activation energy of curing process. This method is based on the combination of tanh fitting for the measured conversion values via least squares minimization technique and linear fitting for the kinetic parameters. Experimentally determined differential scanning calorimetry (DSC) data sets for an epoxy resin functionalized by single wall carbon nanotubes are used for the verification of the proposed method. The results obtained from the proposed algorithm are also compared with the methods reported in the literature.
\end{abstract}

Keywords: Computational material science, Numeical methods, Numerical simulation.

\section{Introduction}

Epoxy resins are extensively used in industry due to their good bonding adhesion to a variety of surfaces and a high chemical and thermal resistance [1],[2]. Since these materials can protect metals against corrosion, they have been employed for coating of internal and external surfaces of pipes [3]. Epoxy resin is mixed with its curing agent and curing process takes place in proper conditions to achieve the desired properties. Knowing the curing kinetics of epoxy can improve and enhance coating quality. There are different techniques to study the curing kinetic of thermosetting resins. Differential scanning calorimetry (DSC) is one of the most common and most accurate methods [4,6] in studying such processes. In the kinetic analysis of a chemical reaction such as resin curing, a mathematical equation between the conversion, conversion rate, and temperature is established [7]. Most of studies employ the model-fitting methods at nonisothermal conditions [8,9]. Although the model-fitting procedure is almost reliable for practical purposes, it does not provide a deep understanding of the detailed reaction mechanism [2]. Although, the most complex kinetics models cannot cover all the complexities of the reactions, to enhance the accuracy, the isoconversional method is recommended for analysis of reaction kinetics [7,10]. Isoconversional methods explain the kinetics of the curing process using several single-stage equations. Each of these equations is related to a specific range of conversion [7] and only one of them will determine the overall kinetics in each region [11]. In isoconversional methods, without considering any specific form of the reaction model, activation energy at a given extent of the conversion can be achieved. By obtaining the dependence of activation energy, kinetics analysis can be performed and a reliable prediction of the kinetics parameters could be provided [12]. Although, for analysis of dynamic data, model-free method is recommended [7], model-fitting method in complex models such as Kamal which can be used for practical applications is reliable as well. To our knowledge, there is no kinetics analysis on a specific epoxy based resin used for pipe lining. Therefore in this study, initially, an advanced model-free method was used. Then, Kamal model was applied to the dynamic data and its parameters were obtained by performing a simple fitting using genetic algorithm. At the end, the ability of both methods to predict the curing behavior of this epoxy resin was investigated. 


\section{Kinetic Model}

Curing reaction of an epoxy resin is an exothermic reaction. In the DSC measurements, the degree of cure $\alpha$ ranges from 0 (completely uncured) to 1 (fully cured) and is defined as follows:

$$
\begin{gathered}
\alpha=\frac{\Delta H_{t}}{\Delta H_{R}} \\
\frac{d \alpha}{d t}=\frac{(d H / d t)_{t}}{\Delta H_{R}}
\end{gathered}
$$

where $H_{t}$ is the amount of heat released in time $t .(d H / d t)_{t}$ is the rate of the heat generation and $\Delta H_{R}$ is the total heat of reaction.

It is commonly accepted in the kinetic analysis of chemical reactions by thermal analysis that any chemical process of reaction will obey a rate law of the form

$$
\frac{d \alpha}{d t}=k(T) f(\alpha)
$$

where $\frac{d \alpha}{d t}$ is the rate of reaction, $k(T)$ is the temperature dependent rate constant, and $f(\alpha)$ corresponds to the reaction model [15]. The temperature dependence of the reaction rate is generally defined through an Arrhenius expression [14]:

$$
k(T)=A \exp \left(-\frac{E}{R T}\right)
$$

where $A$ is the pre-exponential factor, $E$ is the activation energy, $R$ refers to the universal gas constant and $T$ corresponds to the absolute temperature. When the process is isothermal, the temperature is constant, but in the nonisothermal process the temperature usually increases according to a constant heating rate

$$
\beta=\frac{d T}{d t}
$$

where $\beta$ is the heating rate. Combining the Eq. (3), (4) and (5) yields a resulting equation for non-isothermal conditions:

$$
\frac{d \alpha}{d T}=\frac{A}{\beta} \exp \left(-\frac{E}{R T}\right) f(\alpha)
$$

Therefore, if the reaction model which describes the data is known, the conversion obtained at a given temperature or heating rate can be predicted using the above equations.

\section{Experimental}

In this study, single-wall carbon nanotubes (SWCNTs) were supplied from Pinhas Inc., Turkey. SWCNTs were manufactured by TUBALL ${ }^{T M}$, Luxembourg. The outer mean diameter of SWCNTs is below 2 nm, and their length is higher than $1 \mu \mathrm{n}$. It contains high quality of SWCNT (G/D ratio > 90) with an iron content of less than $15 \%$. The resin system used consisted of solvent-type epoxy F- RES21. The viscosity of the resin is 400-650 mPa.s at $25^{\circ} \mathrm{C} .800$ tex carbon fiber (A-49 filaments) were provided from DowAksa Inc., Turkey, and used as reinforcing constituent.

Temperature variations during curing determine the degree of cure of the prepreg system. The degree of curing is highly dependent on the heat of reaction. The results of the DSC experiments are heat flux curves with respect to time and temperature. Samples consisting of 3-6 mg mixtures were placed in aluminum pans. and $50 \mathrm{~mL} / \mathrm{min}$ of $\mathrm{N} 2 \mathrm{purge}$ in DSC 50. Dynamic scans for each sample were performed at different heating rates $2.5,5,10$ and $20^{\circ} \mathrm{C} / \mathrm{min}$ ) from room 
temperature up to $250{ }^{\circ} \mathrm{C}$ in a N2 atmosphere. The upward peaks formed in the DSC thermograms show that the reaction is exothermic.

\section{A new numerical approach: Nonlinear fitting}

Suppose that the experimental data $T=\left[a_{1}, a_{2} \ldots, a_{n}\right]$ and $\alpha=\left[b_{1}, b_{2}, \ldots, b_{n}\right]$ temperature and conversion are given. Consider the activation function as follows:

$$
\alpha(T)=A\left(\tanh \left(B\left(T_{n}-C\right)-3\right)+1\right)
$$

where $A, B$ and $C$ are unknowns. We want to fit a function which has a form in Eq.(7). For this purpose, the nonlinear least square method is used.

\subsection{Nonlinear least-squares data fitting}

In this section we will explain nonlinear least square briefly. Error between the experimental result and and activation function is defined by the following function

$$
E(A, B, C)=\sum_{n=1}^{N}\left(y_{n}-A\left(\tanh \left(B\left(T_{n}-C\right)-3\right)+1\right)\right)^{2}
$$

Requirement of the nonlinear least square procedure is to find a minimum of error function. To find minimum, we set

$$
\frac{\partial E}{\partial A}=0, \quad \frac{\partial E}{\partial B}=0, \quad \frac{\partial E}{\partial C}=0
$$

Partial derivative of $E(A, B, C)$ with respect to $A, B$ and $C$ are

$$
\begin{aligned}
& \frac{\partial E}{\partial A}=\sum_{n=1}^{N} 2\left(-\tanh \left(B\left(T_{n}-C\right)-3\right)\left(y_{n}-A\left(\tanh \left(B\left(T_{n}-C\right)-3\right)+1\right)\right),\right. \\
& \frac{\partial E}{\partial B}=\sum_{n=1}^{N} 2 A B\left(\operatorname{sech}\left(B\left(T_{n}-C\right)-3\right)^{2}\right)\left(y_{n}-A\left(\tanh \left(c_{1}\left(T_{n}-c_{2}\right)-3\right)+1\right)\right), \\
& \frac{\partial E}{\partial C}=\sum_{n=1}^{N}-2 A B\left(\operatorname{sech}\left(B\left(T_{n}-C\right)-3\right)^{2}\right)\left(y_{n}-A\left(\tanh \left(B\left(T_{n}-C\right)-3\right)+1\right)\right) .
\end{aligned}
$$

Combining (9) and (10), we obtain system of nonlinear equation as follows:

$$
\begin{aligned}
0= & \sum_{n=1}^{N}\left(-2 \tanh \left(B\left(T_{n}-C\right)-3\right) y_{n}+2 A \tanh \left(B\left(T_{n}-C\right)-3\right)+2 A \tanh \left(B\left(T_{n}-C\right)-3\right)^{2}\right) \\
0= & \sum_{n=1}^{N}\left(2 A B\left(\operatorname{sech}\left(B\left(T_{n}-C\right)-3\right)^{2} y_{n}-2 A B \tanh \left(B\left(T_{n}-C\right)-3\right) \operatorname{sech}\left(B\left(T_{n}-C\right)-3\right)^{2}-2 A B \operatorname{sech}\left(B\left(T_{n}-C\right)-3\right)^{2}\right)\right. \\
0= & \sum_{n=1}^{N}\left(-2 A B \operatorname{sech}\left(B\left(T_{n}-C\right)-3\right)^{2} y_{n}+2 A B \operatorname{sech}\left(B\left(T_{n}-C\right)-3\right)^{2} \tanh \left(B\left(T_{n}-C\right)-3\right)+2 A B \operatorname{sech}\left(B\left(T_{n}-C\right)-3\right)^{2}\right)
\end{aligned}
$$

When the partial derivative in Eq. (11), (12) and (13) are set equal to zero, the nonlinear system of equations are obtained. We use the Newton Method find the $A, B$ and $C$. Iterative formula of Newton Method for nonlinear systems is given as 
follows:

$$
z^{k+1}=z^{k}-J^{-1}\left(z^{k}\right)\left(\begin{array}{c}
F\left(z^{k}\right) \\
G\left(z^{k}\right) \\
S\left(z^{k}\right)
\end{array}\right)
$$

where $z^{k}=\left(\begin{array}{c}A^{k} \\ B^{k} \\ C^{k}\end{array}\right)$ and $J^{-1}$ is the inverse of the of the Jacobian matrix,

$$
J(z)=\left(\begin{array}{ccc}
\frac{\partial E}{\partial A} & \frac{\partial E}{\partial B} & \frac{\partial E}{\partial C} \\
\vdots & \ddots & \vdots
\end{array}\right)
$$

To start the Newton's iterative method, an initial condition is needed. For convergent $A, B, C$ values we choose appropriate initial condition, $z^{0}=\left(\begin{array}{l}0 \\ 0 \\ 0\end{array}\right)$.

For calculations, Matlab solver is used. Finally we obtain the unknown as $A=0.5, B=0.0765, C=358.3126$

Fig.1 shows the experimental conversion $\alpha(T)$ values and least square approximation of $\alpha(T)$ the activation function.

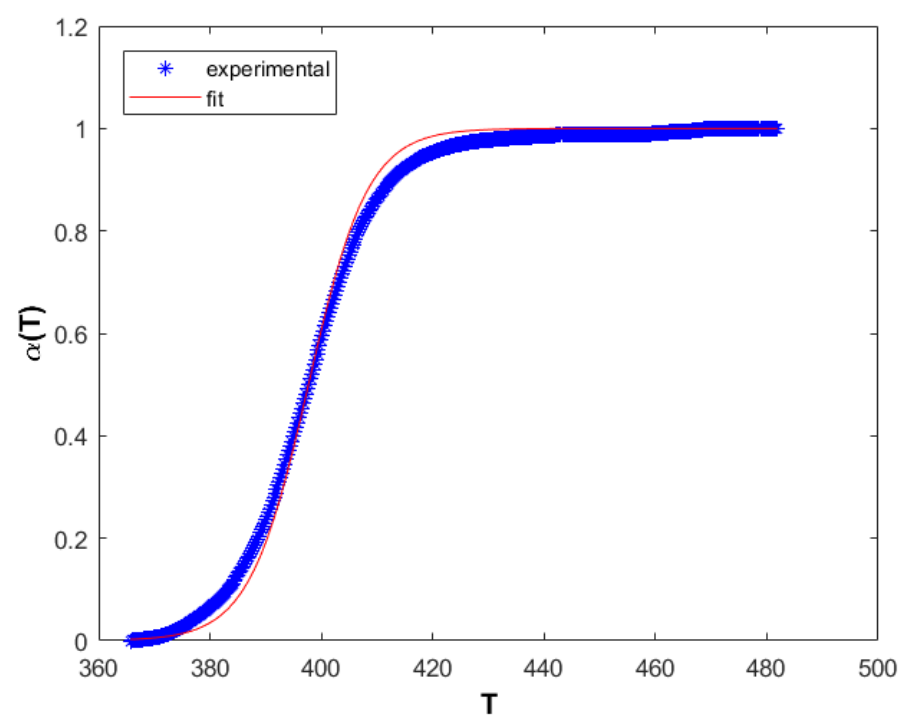

Fig. 1: Temperature and conversion $(\alpha)$ values are plotted from experimental data and activation function which is used fitting.

\subsection{Data linearization technique}

In this section, we will seek values of pre-experimental factor $A$ and activation energy $E$ which are given in Eq.(6). In Eq.(6), there is 4 unknows and they are $A, E, \alpha$ and $f(\alpha)$.

$\alpha(T)$ is obtained in previous section approximately. In the literature there are different choice of $f(\alpha)$. For the model most suitable choice is

$$
f(\alpha)=\alpha^{m}(1-\alpha)^{n}
$$


where $m, n$ are pozitif real numbers. As a result, there are only two unknowns $A$ and $E$ in Eq.(6). To find these unknowns, data linearization technique is used.

The first step is to take the logarithm of both sides of the Eq.(6)

$$
\ln \left(\frac{\beta}{f(\alpha)}\right)+\ln \left(\frac{d \alpha}{d T}\right)=\ln (A)-\frac{E}{R T}
$$

This results in a linear relation between the new values $X$ and $Y$ :

$$
Y=\bar{A}+E X
$$

where

$$
\begin{aligned}
& Y=\ln \left(\frac{\beta}{f(\alpha)}\right)+\ln \left(\frac{d \alpha}{d T}\right), \\
& \bar{A}=\ln (A), \\
& X=-\frac{1}{R T} .
\end{aligned}
$$

To calculate this unknowns, linear fitting method is used. In Table 2, $E$ and $\bar{A}$ values are given for different samples.

\begin{tabular}{|l|l|l|l|l||l||l|}
\hline Sample No & $\beta$ & $A$ & $B$ & $C$ & $E$ & $\ln (A)$ \\
\hline 1 & 2.5 & 0.5 & 0.0765 & 358.3126 & 89.8749 & 22.2212 \\
1 & 5 & 0.5 & 0.0767 & 367.8309 & 85.5814 & 20.9993 \\
1 & 10 & 0.5 & 0.0608 & 368.3936 & 73.3774 & 17.3604 \\
1 & 20 & 0.5 & 0.0564 & 377.0870 & 70.3343 & 16.4869 \\
\hline 2 & 2.5 & 0.5 & 0.0732 & 356.5693 & 82.7869 & 20.0338 \\
2 & 5 & 0.5 & 0.0619 & 359.3702 & 71.0760 & 16.4988 \\
2 & 10 & 0.5 & 0.0558 & 365.7544 & 66.7037 & 15.3149 \\
2 & 20 & 0.5 & 0.0509 & 374.5726 & 69.8242 & 16.2947 \\
\hline 3 & 2.5 & 0.5 & 0.0669 & 352.6327 & 73.1902 & 17.1211 \\
3 & 5 & 0.5 & 0.0627 & 358.7051 & 68.1392 & 15.6939 \\
3 & 10 & 0.5 & 0.0545 & 364.2501 & 63.2666 & 14.2895 \\
3 & 20 & 0.5 & 0.0515 & 375.6366 & 64.49896 & 14.6932 \\
\hline 4 & 2.5 & 0.5 & 0.0651 & 353.4174 & 73.7206 & 17.7973 \\
4 & 5 & 0.5 & 0.0616 & 361.2695 & 76.1108 & 17.9529 \\
4 & 10 & 0.5 & 0.0596 & 369.6381 & 73.5011 & 17.3437 \\
4 & 20 & 0.5 & 0.0561 & 380.6521 & 73.1449 & 15.7400 \\
\hline
\end{tabular}

Table 1: $A, B, C$ obtain from nonlinear fitting and Data Linearization Technique provide getting $E, \ln (A)$ for different $\beta$.

\begin{tabular}{|l|l|l|l|l|l|l|}
\hline \multirow{2}{*}{$\begin{array}{l}\text { Sample } \\
\text { No }\end{array}$} & \multicolumn{2}{|c|}{ Kissinger } & \multicolumn{2}{|c|}{ KAS* } & \multicolumn{2}{c|}{ GMN } \\
\cline { 2 - 7 } & $\mathrm{E}$ & $\ln (A)$ & $\mathrm{E}$ & $\ln (A)$ & $\mathrm{E}$ & $\ln (A)$ \\
\hline 1 & 73.6 & 13.6 & $75.5-61.5$ & $15.2-10.38$ & 73.3774 & 17.3604 \\
2 & 66.3 & 11.3 & $69.0-54.3$ & $13.3-8.2$ & 66.7037 & 15.3149 \\
3 & 68.1 & 11.8 & $61.1-51.6$ & $10.6-7.8$ & 63.2666 & 14.2895 \\
4 & 70.8 & 12.6 & $64.9-68.6$ & $11.6-10.6$ & 73.1449 & 15.7400 \\
\hline
\end{tabular}

Table 2: Comparison of the optimum values of GMN method to with Kissinger and KAS* method. 
Kissinger method gives average of activation energy for whole process containing all $\alpha$ values.

*The KAS method is an isoconversional method and changes depending on $\alpha$. The range given in the table shows the values corresponding to the $\alpha$ value starting from 0.1 and reaching the value 0.9 with 0.05 increments.

\section{Result and discussion}

In this study, the non-isothermal curing behavior of an epoxy resin was studied by differential scanning calorimetry. Kinetic analysis was performed by a new mathematical approach based on the nonlinear least square fitting a tanh function and lumbarization method. This new algorithm is called as a GMN method. Experimentally determined differential scanning calorimetry (DSC) data sets for an epoxy resin functionalized by single wall carbon nanotubes are used for the verification of GMN method. The results obtained from the GMN algorithm are also compared with the methods reported in the literature. Finally, GMN algorithm was in good agreement with experimental data for calculation of the kinetic parameters.

\section{Competing interests}

The authors declare that they have no competing interests.

\section{Authors' contributions}

All authors have contributed to all parts of the article. All authors read and approved the final manuscript.

\section{References}

[1] F. - X. Perrin, T. M. H. Nguyen, J.-L. Vernet, Modeling the cure of an epoxy-amine resin with bisphenola as an external catalyst, Macromolecular Chemistry and Physics 208 (1) (2007) 55-67.

[2] J. Wan, Z.-Y. Bu, C.-J. Xu, H. Fan, B.-G. Li, Model-fitting and model-free nonisothermal curingkinetics of epoxy resin with a low-volatile five-armed starlike aliphatic polyamine, Thermochimica acta525 (1-2) (2011) 31-39.

[3] K. Tsuchida, J. P. Bell, A new epoxy/episulfide resin system for coating applications: curing mechanismand properties, International journal of adhesion and adhesives 20 (6) (2000) 449-456.

[4] W.-Y. Chen, Y.-Z. Wang, F.-C. Chang, Study on curing kinetics and curing mechanism of epoxy resinbased on diglycidyl ether of bisphenol a and melamine phosphate, Journal of applied polymer science92 (2) (2004) 892-900.

[5] H. Xie, B. Liu, H. Yang, Z. Wang, J. Shen, R. Cheng, Thermal characterization of carbon-nanofiber-reinforced tetraglycidyl-4, 4-diaminodiphenylmethane/4, 4-diaminodiphenylsulfone epoxy composites,Journal of applied polymer science 100 (1) (2006) 295-298.

[6] Q. Y. Liu, J. B. Chen, S. M. Liu, J. Q. Zhao, Dynamic cure kinetics of epoxy resins using an amine-containing borate as a latent hardener, Polymer international 61 (6) (2012) 959-965.

[7] S. Vyazovkin, A. K. Burnham, J. M. Criado, L. A. Perez-Maqueda, C. Popescu, N. Sbirrazzuoli, Ictackinetics committee recommendations for performing kinetic computations on thermal analysis data,Thermochimica acta 520 (1-2) (2011) 1-19.

[8] F. Boey, W. Qiang, Experimental modeling of the cure kinetics of an epoxy-hexaanhydro-4-methylphthalicanhydride (mhhpa) system, Polymer 41 (6) (2000) 2081-2094.

[9] J.Sest ak, G. Berggren, Study of the kinetics of the mechanism of solid-state reactions at increasingtemperatures, Thermochimica Acta 3 (1) (1971) 1-12. 
[10] S. Vyazovkin, A unified approach to kinetic processing of nonisothermal data, International journal ofchemical kinetics 28 (2) (1996) 95-101.

[11] M. Vafayan, M. H. Beheshty, M. H. R. Ghoreishy, H. Abedini, Advanced integral isoconversionalanalysis for evaluating and predicting the kinetic parameters of the curing reaction of epoxy prepreg,Thermochimica Acta 557 (2013) 37-43.

[12] S. Vyazovkin, N. Sbirrazzuoli, Isoconversional kinetic analysis of thermally stimulated processes inpolymers, Macromolecular Rapid Communications 27 (18) (2006) 1515-1532.8

[13] H. Cai, P. Li, G. Sui, Y. Yu, G. Li, X. Yang, S. Ryu, Curing kinetics study of epoxy resin/flexible aminetoughness systems by dynamic and isothermal dsc, Thermochimica Acta 473 (1-2) (2008) 101-105.

[14] M. E. Brown, Introduction to thermal analysis: techniques and applications, Vol. 1, Springer Science \& Business Media, 2001.

[15] M. E. Brown, D. Dollimore, A. K. Galwey, Reactions in the solid state, Elsevier, 1980.

[16] Q. Wang, T. He, P. Xia, T. Chen, B. Huang, Cure processing modeling and cure cycle simulationof epoxy-terminated poly (phenylene ether ketone). ii. chemorheological modeling, Journal of appliedpolymer science 66 (4) (1997) 799-808.

[17] S. Vyazovkin, D. Dollimore, Linear and nonlinear procedures in isoconversional computations of theactivation energy of nonisothermal reactions in solids, Journal of chemical information and computersciences 36 (1) (1996) 42-45.

[18] S. Vyazovkin, Evaluation of activation energy of thermally stimulated solid-state reactions under arbi-trary variation of temperature, Journal of computational chemistry 18 (3) (1997) 393-402.

[19] S. Vyazovkin, Model-free kinetics: staying free of multiplying entities without necessity, Journal ofThermal Analysis and Calorimetry 83 (1) (2006) 45-51.

[20] M. Kamal, S. Sourour, Kinetics and thermal characterization of thermoset cure, Polymer Engineering \& Science 13 (1) (1973) $59-64$

[21] J. Lee, H. Choi, M. Shim, S. Kim, Effects of ctbn on the cure characteristics of dgeba/mda/pge-acamsystem, Materials chemistry and physics 52 (3) (1998) 272-276.

[22] C. Zhang, W. K. Binienda, L. Zeng, X. Ye, S. Chen, Kinetic study of the novolac resin curing processusing model fitting and model-free methods, Thermochimica acta 523 (1-2) (2011) 63-69.

[23] D. Shin, H. Hahn, A consistent cure kinetic model for as4/3502 graphite/epoxy, Composites Part A:applied science and manufacturing 31 (9) (2000) 991-999.

[24] G. He, B. Riedl, A. A It-Kadi, Model-free kinetics: Curing behavior of phenol formaldehyde resins bydifferential scanning calorimetry, Journal of applied polymer science 87 (3) (2003) 433-440.

[25] S. Vyazovkin, C. A. Wight, Model-free and model-fitting approaches to kinetic analysis of isothermaland nonisothermal data, Thermochimica acta 340 (1999) 53-68. 